\title{
Care Integration Decreases Hospitalization Costs: Retrospective Cohort Study of Palliative Care Consult Service for Hospital Patients With Metastatic Cancer
}

\section{Antal Tamás Zemplenyi ( $\square$ zemplenyi.antal@pte.hu )}

Syreon Research Institute; University of Pécs Faculty of Pharmacy https://orcid.org/0000-0002-01770264

\section{Ágnes Csikós}

Institute of Primary Health Care, University of Pécs Medical Shool

\section{Petra Fadgyas-Freyler}

Strategic Analysis Department, National Health Insurance Fund, Budapest

\section{Marcell Csanádi}

Syreon Research Institute

\section{Zoltán Kaló}

Center for Health Technology Assessment, Semmelweis University; Syreon Research Institute Éva Pozsgai

Institute of Primary Health Care, University of Pécs Medical School

\section{Maureen Rutten-van Mölken}

School of Health Policy and Management, Erasmus University Rotterdam

János György Pitter

Syreon Research Institute

\section{Research article}

Keywords: metastatic cancer, palliative care consult service, deaths, prognosis

Posted Date: August 25th, 2020

DOl: https://doi.org/10.21203/rs.3.rs-41113/v2

License: (9) (1) This work is licensed under a Creative Commons Attribution 4.0 International License. Read Full License 
The authors have withdrawn this preprint from Research Square 